\title{
Modeling the Impact of Cell Type and Substrate Stiffness on Cell Traction
}

\author{
Srikanth Raghavan, Aravind R. Rammohan, Martial Hervy \\ Science \& Technology Division, Corning Incorporated, Corning, USA \\ Email: raghavans@corning.com
}

Received June 18, 2013; revised July 26, 2013; accepted September 1, 2013

Copyright (C) 2013 Srikanth Raghavan et al. This is an open access article distributed under the Creative Commons Attribution License, which permits unrestricted use, distribution, and reproduction in any medium, provided the original work is properly cited.

\begin{abstract}
We propose a mathematical model to suggest a unified explanation behind the observation that some cell types tend to spread more efficiently on stiff substrates and are able to adapt their internal stiffness to the external stiffness. Our model also offers an explanation regarding the dependence of cell spreading on cell type. We show that our model for stiffness adaptation is in good agreement with experimental data. We also apply our model to calculate the energy of traction on bulk substrates as well as thin coatings, thereby extracting estimates of critical coating thickness as a function of cell type and coating bulk modulus.
\end{abstract}

Keywords: Cell-Matrix Adhesion; Extracellular Matrix; Polyacrylamide Gels; Elasticity Theory; Mechanotransduction; Stem Cell; Modeling

\section{Introduction}

Recent work suggests that the mechanical properties of a cell's microenvironment can have as great an impact on cell structure and function as soluble stimuli and cell-cell contacts [1-11]. The intracellular and extracellular responses to mechanical forces depend both on the material properties of the cell itself and the properties of the surface or matrix to which it is attached [12-15].

While the responses are specific to cell type $[3,15,16]$, there is a clear dependence of cell behavior on substrate rigidity. Specifically, cellular shape, size, and extent of spreading are determined by a specific range of rigidity values that depend on the tissue type from which the cells are derived. For example, certain types of cells grown on stiff substrates exhibit a more spread phenoltype [1], upregulate the expression of integrins [16], assemble actin stress fibers [17], modify the properties, form their substrate adhesions [1,18,19], and activate signaling pathways characteristic of contractility $[18,20,21]$ which eventually modulate cellular function. Hence, cells are able to sense the variation in substrate rigidity and certain types of cells on stiffer substrates which have less rounded morphologies, and are more likely to extend into branched morphologies than the same cells on softer substrates [1,2,7,9,16,22-27]. For example, when cultured on soft polyacrylamide (PA) coatings, normal rat kidney (NRK) epithelial cells [1],
3T3 fibro-blasts [1,16,23,25], endothelial cells [16], smooth muscle cells [7,26], and mesenchymal stem cells (MSCs) [2] have exhibited distinctly more developed focal adhesions and stable morphologies on stiffer substrates.

As cells alter their morphology and downstream behavior when grown on substrates with different rigidity, it is natural to expect that the internal cytoskeletal assembly will change accordingly.

Correspondingly, the internal stresses and the cell stiffness might be expected to change in response to the rigidity of the substrate. Experiments performed on fibroblasts cultured on fibronectin-coated PA gels of varying rigidity [25,28] and alveolar macrophages cultured on collagen-coated PA gels of varying rigidity [29] have shown that cells do, in fact, alter their internal stiffness in response to the substrate stiffness. As described in [30], cell adaptation to substrate rigidity and modulation of internal cell stiffness involve rearrangements of the cytoskeleton and actin dynamics.

Finally, Evans et al. [31] have shown that embryonic stem cells (ESCs) spread and grew more on stiffer substrates. Furthermore, the osteogenic differentiation of ESCs was enhanced on stiff substrates compared to soft substrates, suggesting that the mechanical environment can play a role in both early and terminal ESC differentiation.

Taken together, these studies show that matrix stiff- 
ness plays a key role in influencing cell morphology and, crucially, downstream stem cell behavior.

Several models have been proposed to explain and describe the influence of substrate stiffness and thickness on the mechanobiology of cells. Building on the classic work by Eshelby [32], Zemel and co-workers have investigated in great detail, the influence of substrate mechanical properties on cell shape, symmetry of cell spreading, and polarization of stress fibers in cells [33-35]. The key role and behavior of cell stiffness and focal adhesions have been explored in detail by Bischofs and Schwarz [12], Schwarz et al. [13,14], Nicolas et al. [36], Shemesh et al. [37], and Besser and Safran [38]. Detailed models that couple biochemistry and cell adhesion mechanics have also been developed [39-41] to give much insight into the problem of mechanosensing. The influence of substrate thickness on cell behavior has also received much attention recently, notably in works by Maloney et al. [23], Merkel et al. [42], and Lin et al. [43] among others.

However, what has been lacking thus far is a simple model that links the energetics of cell traction on bulk substrates with the influence of substrate thickness on cell traction and that can thus guide the optimal design of substrates to enable efficient cell traction.

In this article, we describe a model that is primarily concerned with the energetics of cell traction and that focuses on three distinct but related observations concerning the connection between cell behavior and the stiffness of the substrate/coating on which cells are cultured:

1) Stiffer substrates encourage more efficient and increased spreading of some cell types [1,2,7,9,16,22-27].

2) As part of the response to the mechanical properties of the substrates, some cell types, e.g. fibroblasts, are able to adapt their internal stiffness to substrate stiffness better than other cell types [25,28].

3) Some cell types (e.g. fibroblasts) spread more on soft, thin gels $[2,23,25,44,45]$ and thus show that they are able to sense the effective stiffness of a thin soft gel attached to much stiffer supports, e.g. glass.

In contrast to earlier detailed models [33-38], our model is primarily concerned with the energy involved in the traction process and hence does not describe cell shape or cell spreading. Our model takes into account the interaction between internal cell stiffness and external stiffness in a simple fashion as described by Schwarz et al. $[13,14]$. Hence, we do not describe either the details of focal adhesions as have been done in [12,36-38] or the role of biochemical coupling to cell mechanics as described in [39-41]. Crucially, however, our model recognizes the fact that the substrate stiffness perceived by the cell can be thickness-dependent and incorporates the models of Maloney et al. [23] and Merkel et al. [42] and links them to the energetics of the traction process.

We further note that cell-material interaction is mediated by multiple focal adhesions in reality and the interaction is a very complex process. Therefore, as a first step, we focus in this paper on describing the energetics of a single focal adhesion complex interacting with a substrate. Hence, throughout this paper, when we talk about cellular traction, it is in the context of the traction associated with a single focal adhesion interacting with a substrate.

\section{Model}

The model in this paper is chiefly concerned with the energetics of cell traction. For the purposes of this paper, we will define traction to be the process by which a cell experiences forces exerted by the underlying substrate through focal adhesions (FAs) and the resultant mechanical response of cells. As a cell experiences forces exerted by the substrate, for it to initiate a mechanical response to these forces, the cell has to invest a certain amount of energy to trigger a complex set of downstream responses that include but are not limited to adhesion, spreading, and motility. Therefore, understanding the energetics of the traction process could allow us to explain differences in downstream cell responses.

Specifically, in designing coatings on surfaces for adherent cells to demonstrate certain functional behavior, the ability to predict differences in cellular traction for different combinations of cell types and underlying coating/substrate stiffness and thickness is very valuable. For example, this understanding can help in answering a specific question such as: when cells of different types are cultured on thin coatings of variable bulk stiffness fixed to stiff substrates, what is the impact of the thickness and bulk stiffness of the coating on the energetics of the traction process in particular? Using our model, we will tackle this question in this paper.

Our model builds upon two existing but separate models: 1) one due to Schwarz et al. $[13,14]$ for substrate rigidity sensing, and 2) the other due to Maloney et al. [23] for substrate thickness sensing, and introduces a heuristic expression describing the behavior of some cell types of adapting their internal stiffness while exhibiting more spread morphologies responding to substrate stiffness. For the sake of completeness, we briefly review these earlier models.

\subsection{Rigidity Sensing Model}

Schwarz et al. [13] proposed a simple two spring system shown in Figure 1. The figure shows an idealized schematic of a two-spring model for rigidity-sensing through cell-matrix contacts at focal adhesion sites. It is assumed that the traction applied by the cell to the matrix at 


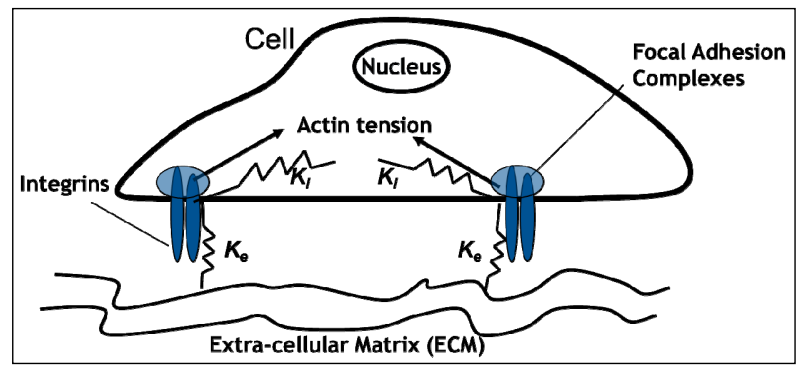

Figure 1. Schematic of two-spring model for rigidity sensing through cell-matrix contacts (figure adapted from $[13,14]$ ). The spring constant $K_{e}$ represents extracellular elasticity as perceived by the cell through the focal adhesion site. The spring constant $K_{i}$ represents the mechanical properties of the intracellular structure.

the focal adhesion takes place through a spring whose spring constant is denoted by $K_{e}$. This spring constant represents the extracellular matrix (ECM) elasticity. Later in this article, we will invoke the treatment by Maloney et al. [23] to make contact between $K_{e}$ and the elastic modulus of the ECM, $E_{\text {ext }}$, perceived by the cell. The external spring is in series with another spring that represents the mechanical properties of the intracellular structure and has a spring constant denoted by $K_{i}$.

Since the internal spring and external spring are in series, the effective spring constant of the system is given by

$$
\frac{1}{K_{e f f}}=\frac{1}{K_{i}}+\frac{1}{K_{e}} .
$$

In the original two-spring model of Schwarz et al. [13], the internal tension in the actin stress fibers is generated by myosin II molecular motors. The system is strained by a cytoskeletal molecular motor represented by a linearized force-velocity relation that is modeled as

$$
v(F)=v_{0}\left(1-\frac{F}{F_{s}}\right)
$$

where $v_{0}$ is the free velocity of the motor and $F_{s}$ is the stall force. Typically the free velocity of the motor is of the order of $v_{0}=10 \mu \mathrm{m} / \mathrm{s}$ and the stall force $F_{s}$ is of the order of few $\mathrm{pN}$ [46]. A preliminary observation, viz. that the effective spring constant is given by Equation (1), immediately tells us that the softer spring determines the mechanical response of the cell in the traction process.

As the cell strains, the molecular motors invest power $v(F) F$ which is derived from the power stored in the springs. Thus, we have

$$
\frac{d}{d t}\left(\frac{F^{2}}{2 K_{\text {eff }}}\right)=v(F)=v_{0}\left(1-\frac{F}{F_{s}}\right) F
$$

where we have used Equation (2). We can integrate
Equation (3) trivially to yield the dynamics of force build-up over time,

$$
F(t)=F_{s}\left(1-e^{-t / t_{k}}\right),
$$

where

$$
t_{K} \equiv \frac{F_{s}}{v_{0} K_{e f f}} .
$$

Thus, larger the effective spring constant $K_{\text {eff }}$, smaller the time constant $t_{K}$, and more efficient the force build-up. We can compute the total energy invested by the cell in the traction process in a straightforward manner,

$$
w=\int_{0}^{\infty} F v(F) \mathrm{d} t=\frac{F_{s}^{2}}{2 K_{e f f}} .
$$

We wish to make it clear that although the force generation in cells in response to external influences is dynamic in nature [13,47-49], our paper will not deal with dynamic, time-dependent traction processes.

\subsection{Stiffness Sensing of Cells on Thin Substrates}

In order to make further progress, we need a connection between the external elastic modulus $E_{\text {ext }}$, and the external spring constant $K_{e}$, of the substrate as perceived by the cellular focal adhesion. Fortunately, the model by Maloney et al. [23] provides precisely such a connection by tracking the displacement of the focal adhesion site and the traction force for both bulk substrates and coatings with finite thickness supported on materials with large elastic modulus. We will first discuss bulk substrates. The model by Maloney et al. [23] treats an idealized circular adhesion site of radius $a$ pulling on a substrate with effective elastic modulus $E_{b u l k}$ and Poisson's ratio $v$ and yields the following relation between $K_{e}$ and $\varepsilon_{\text {ext }}$,

$$
K_{e}=\frac{\pi \varepsilon_{\text {exta }}}{(1+v)(2-v)}
$$

Throughout this article, we will set Poisson's ratio, $v$ to 0.5 , and assume a focal adhesion size of $a=1.0 \mu \mathrm{m}$ consistent with the values used in [23].

As observed by $[2,23,24,44,45]$ among others, when some cells are cultured on soft, thin substrates that are attached to stiff supports, these cells are able to "feel" the effective stiffness of the composite substratum. Thus cells are able to spread far greater than what the soft substrates alone would suggest. Maloney et al. [23] modeled this situation using a simple model based on linear elas-

${ }^{1}$ We note here that whereas for bulk substrates, $\mathcal{E}_{\text {ext }}=E_{\text {bulk }}$, for thin substrates supported on materials with large elastic modulus, $\mathcal{E}_{\text {ext }}$ is modified by a thickness-dependent function as described in [23]. We will treat this case later in this section. 
ticity. One key parameter that governs cell behavior is the ratio of substrate or coating thickness, $h$, to focal adhesion size, $a$. The effective stiffness perceived by the cell is given by

$$
\varepsilon_{\text {ext }}(h / a)=\frac{E_{\text {bulk }}}{F\left(\frac{h}{a}\right)}
$$

where $F(h / a)$ is a thickness dependent normalized function that can be approximated very well for our purposes as [23]:

$$
\begin{aligned}
F(h / a) \cong & 1-\frac{1}{2 a(2-v)} \times \\
& \left(\frac{h^{2}}{\sqrt{h^{2}+a^{2}}}+(4-2 v) \sqrt{h^{2}+a^{2}}-(5-2 v) h\right)^{.}
\end{aligned}
$$

\subsection{Stiffness Adaptation of Cells}

We have thus far described earlier models due to Schwarz et al. $[13,14]$ for substrate thickness sensing. We will now discuss how we extend these models to include the phenomenon of stiffness adaptation of cells. As described by Solon et al. [25] and Tee et al. [28], some cell types have a tendency to increase their internal cell stiffness while adapting to the stiffness of the substrate on which the cells are cultured. The arguments laid out in Section 2.1 show that it is the effective stiffness perceived by the cells that determines the energy of traction. To that end, it is reasonable to suggest that when cultured on substrates with a large stiffness, it is energetically favorable for cells to raise their own internal stiffness so as to lower the overall energy of traction $w$ as defined in Equation (6). A related observation is that whereas some cell types are able to spread more on stiff substrates, others are relatively insensitive to substrate stiffness [17]. We capture this adaptation of cell stiffness and dependence on cell type as a relationship wherein cells can modify their internal spring constant $K_{i}$, up to a certain maximum, $K_{i-\max }$ that would presumably be dependent on cell type through a heuristic expression:

$$
K_{i}\left(K_{e}\right)=K_{i-\max }\left(1-e^{-K_{e} / K_{i-\max }}\right) .
$$

In order to compare our proposed heuristic model with experiment, we rewrite Equation (10) in terms of elastic modulus as:

$$
\varepsilon_{i}\left(\varepsilon_{\text {ext }}\right)=\varepsilon_{i-\max }\left(1-e^{-\varepsilon_{\text {ext }} / \varepsilon_{i-\max }}\right)
$$

We display in Figure 2(a) the comparison between the experiments of Solon et al. [25] on fibroblasts cultured on fibronectin-coated polyacrylamide gels of variable stiffness and fibronectin-coated glass and the model shown in Equation (11). An estimate of $\mathcal{E}_{i-\max } \sim 5.5 \mathrm{kPa}$

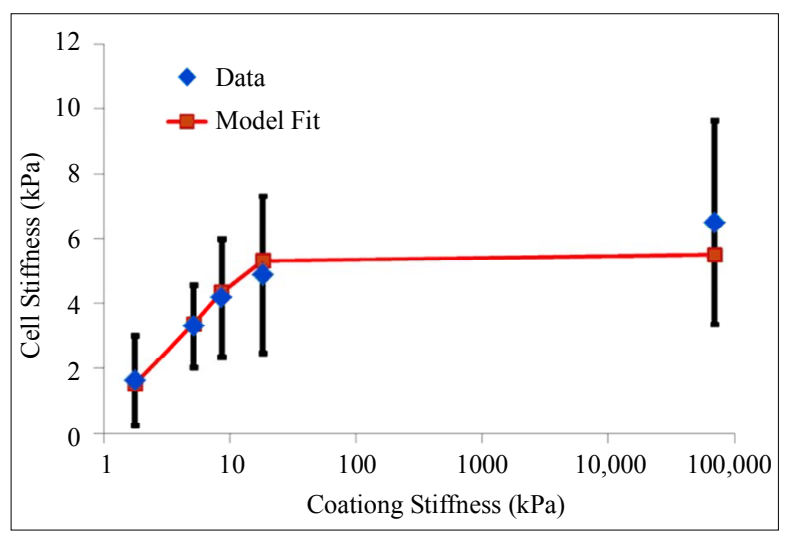

(a)

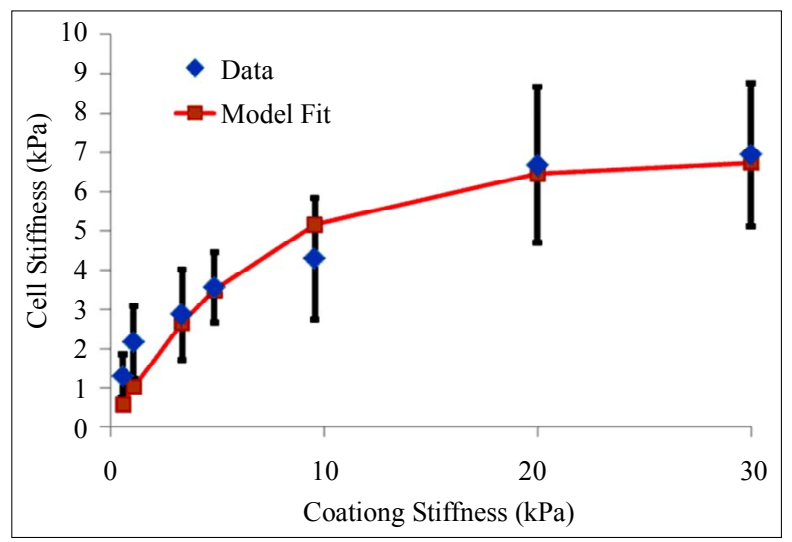

(b)

Figure 2. Comparison between stiffness adaptation model depicted by blue diamonds and line and experiments (a) by Solon et al. [25] depicted by red squares and (b) by Tee et al. [28] depicted by red squares.

(or equivalently $K_{i-\max } \sim 7.7 \mathrm{pN} / \mathrm{nm}$ ) gives the best fit between the model and experiment.

In Figure 2(b), we plot the comparison between the experiments of Tee et al. [28] on human MSCs (hMSCs) cultured on fibronectin-coated polyacrylamide gels of variable stiffness and the model shown in Equation (11). An estimate of $\mathcal{E}_{i-\max } \sim 6.8 \mathrm{kPa}$ (or equivalently $K_{i-\max } \sim 9.5 \mathrm{pN} / \mathrm{nm}$ ) gives the best fit between the model and experiment.

Equation (10) (or its analogous Equation (11)) naturally gives us two limits: for $K_{e} \ll K_{i-\max }, K_{i} \sim K_{e}$, and for $K_{e} \gg K_{i-\max }, K_{i} \sim K_{i-\max }$. Equation (10) (or its analogous Equation (11)) also suggests that cell types with larger $K_{i-\max }$ have greater intrinsic ability to gain traction by greater lowering of traction energy. This suggestion is supported by experiments $[16,25,28]$ that show that some cell types are more able than other cell types to modify their internal stiffness and thereby spread more easily on stiff substrates.

We note here that our heuristic model for cell adaptation presented in Equations (10) and (11) is by no means 
unique. Other possible models could be chosen with similar limiting forms as the one that our model gives. We have chosen our model so that it is simple enough and yet give the right adaptation behavior. Clearly, a combination of more detailed experiments and models are required to give a more accurate adaptation model. We further recognize that stiffness adaptation involves a complex interplay between two distinct aspects of the mechanochemical transduction process of cell surface receptors: 1) recognition of differences in substrate rigidity and 2) initiation of intracellular changes resulting in cytoskeletal reorganization. Combining Equations (1), (6) and (10), we obtain the total system energy invested by the cell during the traction process:

$$
w=\frac{F_{s}^{2}}{2 K_{\text {eff }}}=\frac{F_{s}^{2}}{2}\left[\frac{1}{K_{e}}+\frac{1}{K_{i-\max }\left(1-e^{-K_{e} / K_{i-\max }}\right)}\right]
$$

Since we are primarily interested in changes in traction energy as a function of substrate properties, we normalize the expression given in Equation (12) with respect to its limiting value at small external stiffness,

$$
\begin{gathered}
w_{0}=\frac{F_{s}^{2}}{K_{e}} \\
w_{\text {norm }}=\frac{w}{w_{0}}
\end{gathered}
$$

Throughout the paper, we will focus on the normalized value of the traction energy as shown in Equation (14). As has been noted earlier in the paper, whereas the force generated by cells increases monotonically and dynamically with substrate rigidity [47-49], we focus in this paper on traction processes involving single focal adhesion complexes. The increase in force generation within cells is likely caused by the recruitment of more proteins and hence an increase in number of focal adhesion complexes.

The expression for traction energy in Equation (12) immediately suggests that systems with larger effective spring constants will lead cells to invest less energy in the traction process and can thus help cells gain traction more easily. This suggestion in turn implies the following:

1) Substrates those are stiffer, either because they have intrinsically larger elastic modulus or because they are thin and attached to stiff supports, will yield larger effective $K_{\text {eff }}$ and thus result in more efficient traction process. This is in line with experimental observations $[1,2$, $7,9,16,22-27]$.

2) It is energetically favorable for cells to raise their internal stiffness to match the stiffness of the ECM as is observed for fibroblasts by Solon et al. [25] and for hMSCs by Tee et al. [28].

\section{Results: Traction Energy of Cells on Bulk Substrates}

We are now in a position to discuss the energetics of the traction process when cells of different types spread on substrates with a range of elastic moduli. In Figure 3, we display the normalized traction energy as a function of substrate coating stiffness. The figure illustrates several important points. First, it is clear that stiffer substrates require less investment of traction energy by the cell. Consequently, such substrates promote better traction. Second, in order to take advantage of the higher stiffness of such substrates, the cells must themselves have the ability to match, i.e. have sufficient internal stiffness $K_{i-\max }$. Since mammalian cells exhibit a wide range of Young's moduli, from as soft as $10 \mathrm{~Pa}$ to as stiff as 100 $\mathrm{kPa}$ [50], we can immediately connect our findings with cells in this range. For instance, cells such as those belonging to neuronal cell type that have $K_{i-\max }=1 \mathrm{pN} / \mathrm{nm}$ are able to lower the traction energy much less compared to their stiffer counterparts. On the other hand, fibroblast cells and hMSCs that have higher stiffness in the range of $K_{i-\max }=5-10 \mathrm{pN} / \mathrm{nm}$ and stiffer cells in the range of $K_{i-\max }=10-20 \mathrm{pN} / \mathrm{nm}$ are able to substantially lower the traction energy when cultured on stiff substrates. This could presumably explain the relative ease of traction of stiff cells such as fibroblasts on stiff substrates.

As is to be expected, the curves in Figure 3 show a distinct cross-over from an initial drop to a plateau-like behavior to ones with the cross-over occurring around $K_{e} \sim K_{i-\max }$.

\section{Results: Traction Energy of Cells on Thin Coatings}

As observed in $[2,23,25,44,45]$ among others, when some cells are cultured on soft, thin substrates that are attached to stiff supports, these cells are able to "feel" the effective stiffness of the composite substratum. Thus cells are able to spread far greater than what the soft sub-

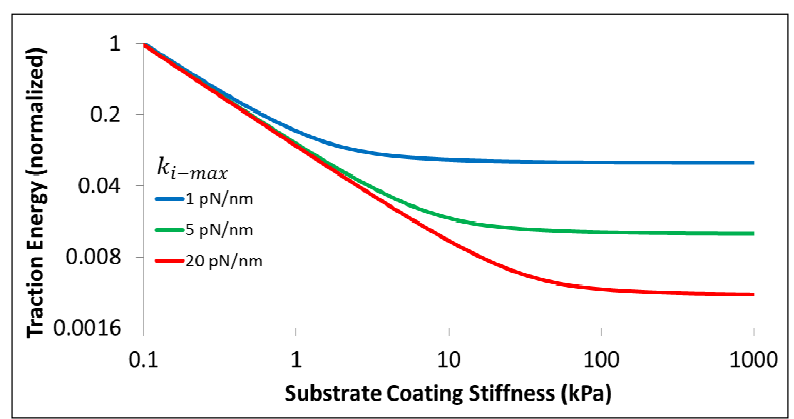

Figure 3. Energetics of traction process for three different cell types, characterized by different values of $K_{i-\max }$, as a function of substrate elastic modulus. 
strates alone would suggest. We will now apply the methods of the earlier Sections 2.1, 2.2, 2.3, and 3 to discuss the energetics of the traction process when cells of different types spread on substrates with fixed or variable intrinsic elastic moduli but different thicknesses. Combining Equations (7)-(12), we can write the energetics of the traction process for cells whose focal adhesion size is given by $a$ on coatings of thickness denoted by $h$ as:

$$
\begin{aligned}
& w\left(\frac{h}{a}\right)=\frac{F_{s}^{2}}{2 K_{e f f}\left(\frac{h}{a}\right)}= \\
& \frac{F_{s}^{2}}{2}\left(\frac{1}{K_{e}(h / a)}+\frac{1}{K_{i-\max }\left[1-e^{-K_{e}\left(\frac{h}{a}\right) / K_{i-\max }}\right]}\right)
\end{aligned}
$$

Again, as before, we are primarily interested in the traction energy normalized with respect to its value at bulk substrates, as given by:

$$
w_{\text {norm }}\left(\frac{h}{a}\right)=\frac{w\left(\frac{h}{a}\right)}{w_{\text {bulk }}} ;\left.w_{\text {bulk }} \equiv w\left(\frac{h}{a}\right)\right|_{h \gg a}
$$

We can now analyze the dependence of the energetics of the traction process on cell type as well as thickness of coating on which the cells are cultured by studying Figure 4.

As the figure shows, thin coatings substantially lower the energy required for the traction process. However, this lowering of energy can be truly beneficial only for cells that have the necessary internal stiffness and cytoskeletal machinery, i.e. sufficient $K_{i-\max }$. For instance, whereas the traction energy is lowered two-fold for cells with $K_{i-\max }=1 \mathrm{pN} / \mathrm{nm}$ for thin substrates compared to their bulk counterpart, stiffer cells with

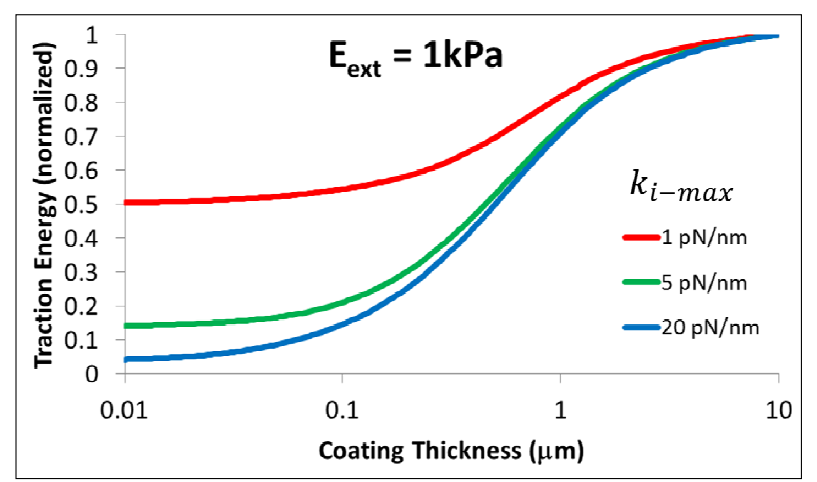

Figure 4. Energetics of traction process for three different cell types, characterized by different values of $K_{i-\max }$, as a function of coating thickness for a coating of bulk elastic modulus of $E_{e x t}=1 \mathrm{kPa}$.
$K_{i-\max }=20 \mathrm{pN} / \mathrm{nm}$ are able to lower their traction energy by more than a factor of 20 .

We can further investigate the reduction of traction energy for thin substrates by studying the reciprocal of the normalized traction energy given by Equation (16) evaluated at a substrate thickness, $h$ and denote this quantity by $M(h)$. We evaluate $M(h)$ at a thickness of $h=0.01 \mu \mathrm{m}$ and display it as a function of cell stiffness and various values of bulk substrate stiffness in Figure 5.

Figure 5 shows clearly that stiff cells are able to significantly lower the traction energy when cultured on thin substrates compared to soft cells. Further, it is clear that those substrates that have low bulk stiffness offer much great ability to influence cell behavior through their thickness than substrates that have high bulk stiffness values. This is reasonable to expect since those substrates that are already stiff in their bulk state are perceived by the cells to become even stiffer and not able to exert much additional influence. On the other hand, substrates that are soft in their bulk state can appear considerably stiffer because of their reduced thickness. Thus, the influence of soft thick substrates can be extended across a wide range of cell types by controlling the thickness alone.

Since the thickness of soft coatings can significantly modulate traction energy, the question of an optimal or critical thickness for a given cell type and function assumes immediate importance for several conceptual as well as practical reasons. First, while the chemistry of the coating can be tuned [1] to achieve the elastic modulus of interest, there is always a danger that the biochemistry could be sufficiently changed so as to modify cell function. Thus, it is sometimes preferable to keep the chemistry intact but modify the effective elastic modulus by changing the thickness of the coating alone as shown by Maloney et al. [23] and described in Section 2.2 in this paper. A second practical reason for choosing an optimal

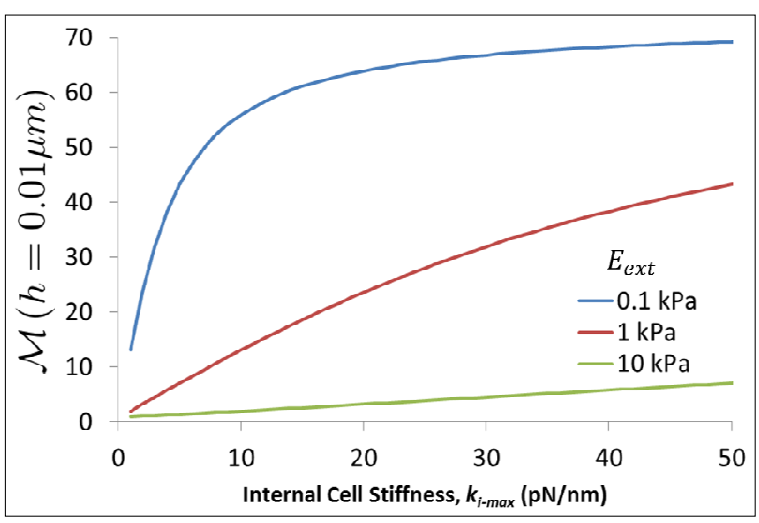

Figure 5. Reciprocal of normalized traction energy evaluated at substrate thickness of $0.01 \mu \mathrm{m}$ as a function of cell stiffness and various values of bulk substrate stiffness. 
or critical thickness stems from the consideration that using substrate coatings that are more than $1 \mu \mathrm{m}$ thick may be undesirable from a processing and imaging point of view. One would thus desire a coating that would be as thin as possible and yet be able to effectively modulate cell function and phenotype.

In the context of the present paper, for the sake of simplicity, we will characterize the energy of traction to be a cell phenotype that can be modulated by coating stiffness and thickness. Further, we will monitor the ratio of the traction energy the cell has to invest for a bulk coating to the traction energy invested by the cell when cultured on a coating of thickness $h$. As Figure 4 shows, this ratio will continue to increase till a threshold value below some critical thickness, $h_{\text {crit }}$ beyond which we can state with some confidence that the coating has ceased to modulate cell function; instead cell function is dictated predominantly by the elastic modulus of the stiff substrate on which the coating is fixed. We plot in Figure 6, the behavior of the critical thickness $h_{\text {crit }}$ as a function of intrinsic cell stiffness $k_{i-\max }$ (i.e., different cell type), where we have fixed the threshold value to be equal to 2 . In Figure 6, different cell types are demarcated using vertical lines; for example, cells in region I correspond to the neuronal cell type whereas cells in region II correspond to fibroblasts and hMSCs. Figure 6 is instructive for it shows that the softest coatings have the greatest potential for modulation of cell function through their thickness alone since they maintain practically uniform behavior across a wide variety of cell types. Coatings that are stiff to begin with cannot confer much additional benefit due to their thickness. Figure 5 also yields specific guidelines on how thick a coating with a given bulk elastic modulus needs to be when culturing a cell type of a given intrinsic stiffness in order that the thin coating is able to modulate cell traction effectively. For example,

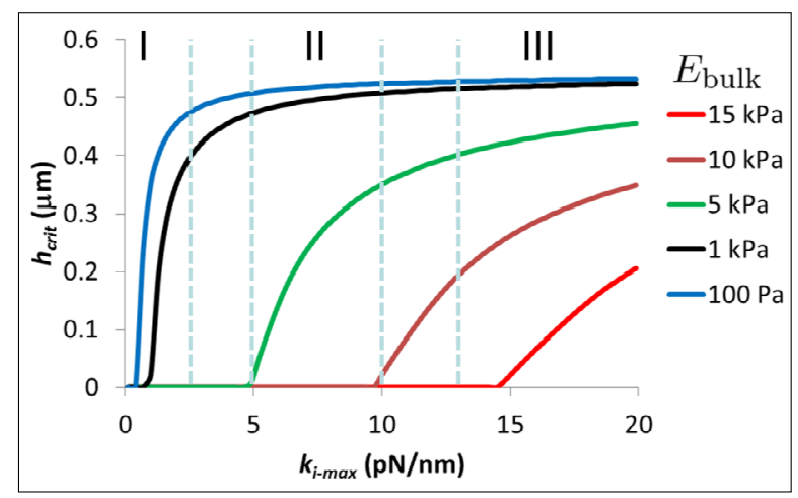

Figure 6. Critical coating thickness $\boldsymbol{h}_{\text {crit }}$ as a function of intrinsic cell stiffness $\boldsymbol{k}_{i \text {-max }}$ corresponding to different cell types for various values of coating bulk modulus. Cells in region I correspond to the neuronal cell type, cells in region II correspond to fibroblasts and hMSCs, and cells in region III correspond to muscle and bone cell types. for cells in the stiffness range of $2-10 \mathrm{pN} / \mathrm{nm}$, coatings with a bulk modulus of $E_{b u l k}=1 \mathrm{kPa}$ need to be in the thickness range of $0.35-0.5 \mu \mathrm{m}$ so that the cells can lower their traction energy by at least a factor of 2 compared to the cells cultured on bulk coatings.

\section{Conclusions}

We have proposed a model that is chiefly concerned with the energetics of cell traction with a focus on the observations that some cell types tend to 1) spread more efficiently on effectively stiff substrates and 2) better adapt their internal stiffness in relation to the external stiffness of substrates. We have built on ideas described in a simple two-spring model for rigidity sensing by $[13,14]$ and a model for substrate thickness sensing by Maloney et al. [23] to show that some cell types can lower the energy required for traction when cultured on stiff, thick substrates or soft, thin substrates. We have also proposed a phenomenological model to match the experiments on stiffness adaptation by Solon et al. [25] and Tee et al. [28]. Furthermore, we have applied our model to calculate the energy of traction on bulk substrates as well as thin coatings and thereby extract estimates of critical coating thickness as a function of cell type and coating bulk modulus. While we emphasize that cells are complex systems in general and the phenomenon of cell mechanosensing in particular is profoundly intricate [51], we believe that simple models such as ours can, nevertheless, provide useful insights into the traction process. We list below specific findings from our work.

1) Using a stiffness matching model we estimate, the maximum cell stiffness for fibroblasts and hMSCs when cultured on polyacrylamide coated glass to be $5.5 \mathrm{kPa}$ and $6.8 \mathrm{kPa}$.

2) Stiffer cells that can raise internal stiffness by saying a factor of 20 with respect to softer cells will require almost 20 fold lesser energy for the traction.

3) Finally, we provide a preliminary phase diagram of coating thickness needed for cells of different internal stiffness to require a change of no more than a factor of 2 in energy invested in the traction process.

\section{REFERENCES}

[1] R. J. Pelham Jr. and Y. L. Wang, "Cell Locomotion and Focal Adhesions are Regulated by Substrate Flexibility," Proceedings of the National Academy of Sciences USA, Vol. 94, No. 25, 1997, pp. 13661-13665. http://dx.doi.org/10.1073/pnas.94.25.13661

[2] A. J. Engler, S. Sen, H. L. Sweeney and D. E. Discher, "Matrix Elasticity Directs Stem Cell Lineage Specification," Cell, Vol. 126, 2006, pp. 677-689. http://dx.doi.org/10.1016/j.cell.2006.06.044

[3] D. E. Discher, P. Janmey and Y. L. Wang, "Tissue Cells Feel and Respond to the Stiffness of Their Substrate," 
Science, Vol. 310, No. 5751, 2005, pp. 1139-1143. http://dx.doi.org/10.1126/science.1116995

[4] A. J. Keung, K. E. Healy, S. Kumar and D. V. Schaffer, "Biophysics and Dynamics of Natural and Engineered Stem Cell Microenvironments," Wiley Interdisciplinary Reviews: Systems Biology and Medicine, Vol. 2, No. 1, 2009, pp. 49-64. http://dx.doi.org/10.1002/wsbm.46

[5] C. S. Chen, M. Mrksich, S. Huang, G. M. Whitesides and D. E. Ingber, "Geometric Control of Cell Life and Death," Science, Vol. 276, No. 5317, 1997, pp. 1425-1428. http://dx.doi.org/10.1126/science.276.5317.1425

[6] A. J. Engler, M. A. Griffin, S. Sen, C. G. Bonnemann, H. L. Sweeney and D. E. Discher, "Myotubes Differentiate Optimally on Substrates with Tissue-Like Stiffness: Pathological Implications for Soft or Stiff Microenvironments," Journal of Cell Biology, Vol. 166, No. 6, 2004, pp. 877887. http://dx.doi.org/10.1083/jcb.200405004

[7] S. R. Peyton and A. J. Putnam, "Extracellular Matrix Rigidity Governs Smooth Cell Motility in a Biphasic Fashion," Journal of Cellular Physiology, Vol. 204, No. 1, 2005, pp. 198-209. http://dx.doi.org/10.1002/jcp.20274

[8] G. Jiang, A. H. Huang, Y. Cai, M. Tanase and M. P. Sheetz, "Rigidity Sensing at the Leading Edge through $\alpha_{v} \beta_{3}$ Integrins and RPTP $\alpha$," Biophysical Journal, Vol. 90, No. 5, 2006, pp. 1804-1809. http://dx.doi.org/10.1529/biophysj.105.072462

[9] W. Guo, M. T. Frey, N. A. Burnham and Y. Wang, "Substrate Rigidity Regulates the Formation and Maintenance of Tissues," Biophysical Journal, Vol. 90, 2006, pp. 22132220. http://dx.doi.org/10.1529/biophysj.105.070144

[10] K. Ghosh, Z. Pan, E. Guan, S. Ge, Y. Liu, T. Nakamura, X. D. Ren, M. Rafailovich and R. A. F. Clark, "Cell Adaptation to a Physiologically Relevant ECM Mimic with Different Viscoelastic Properties," Biomaterials, Vol. 28, No. 4, 2007, pp. 671-679.

http://dx.doi.org/10.1016/j.biomaterials.2006.09.038

[11] B. Geiger and A. Bershadsky, "Exploring the Neighborhood: Adhesion-Coupled Cell Mechanosensors," Cell, Vol. 110, No. 2, 2002, pp. 139-142. http://dx.doi.org/10.1016/S0092-8674(02)00831-0

[12] I. B. Bischofs and U. S. Schwarz, "Cell Organization in Soft Media Due to Active Mechanosensing," Proceedings of the National Academy of Sciences USA, Vol. 100, No. 16, 2003, pp. 9274-9279.

http://dx.doi.org/10.1073/pnas.1233544100

[13] U. S. Schwarz, T. Erdmann and I. B. Bischofs, "Focal Adhesions as Mechanosensors: The Two-Spring Model," BioSystems, Vol. 83, 2006, pp. 225-232. http://dx.doi.org/10.1016/j.biosystems.2005.05.019

[14] U. S. Schwarz, "Soft Matters in Cell Adhesion," Soft Matter, Vol. 3, No. 3, 2007, pp. 263-266. http://dx.doi.org/10.1039/b606409d

[15] P. C. Georges and P. A. Janmey, "Cell Type-Specific Response to Growth on Soft Materials," Journal of Applied Physiology, Vol. 98, No. 4, 2005, pp. 1547-1553. http://dx.doi.org/10.1152/japplphysiol.01121.2004

[16] T. Yeung, P. C. Georges, L. A. Flanagan, B. Marg, M. Ortiz, M. Funaki, N. Zahir, W. Ming, V. Weaver and P. A.
Janmey, "Effects of Substrate Stiffness on Cell Morphology, Cytoskeletal Structure, and Adhesion," Cell Motility and the Cytoskeleton, Vol. 60, No. 1, 2005, pp. 24-34. http://dx.doi.org/10.1002/cm.20041

[17] P. C. Georges, W. J. Miller, D. F. Meaney, E. S. Sawyer and P. A. Janmey, "Matrices with Compliance Comparable to that of Brain Tissue Select Neuronal over Glial Growth in Mixed Cortical Cultures," Biophysical Journal, Vol. 90, No. 8, 2006, pp. 3012-3018. http://dx.doi.org/10.1529/biophysj.105.073114

[18] M. J. Paszek, N. Zahir, K. R. Johnson, J. N. Lakins, G. I. Rozenberg, A. Gefen, C. A. Reinhart-King, S. S. Margulies, M. Dembo, D. Boettiger, D. A. Hammer and V. M. Weaver, "Tensional Homeostasis and the Malignant Phenotype," Cancer Cell, Vol. 8, No. 3, 2005, pp. 241-254. http://dx.doi.org/10.1016/j.ccr.2005.08.010

[19] A. Engler, L. Bacakova, C. Newman, A. Hategan, M. Griffin and D. E. Discher, "Substrate Compliance versus Ligand Density in Cells on Gel Responses," Biophysical Journal, Vol. 86, No. 1, 2004, pp. 617-628. http://dx.doi.org/10.1016/S0006-3495(04)74140-5

[20] J. Pourati, A. Maniotis, D. Spiegel, J. L. Schaffer, J. P. Butler, J. J. Fredberg, D. E. Ingber, D. Stamenovic and N. Wang, "Is Cytoskeletal Tension a Major Determinant of Cell Deformability in Adherent Endothelial Cells?" American Journal of Physiology Cell Physiology, Vol. 274 1998, pp. C1283-C1289.

[21] M. Chrzanowkska-Wodnicka and K. Burridge, "RhoStimulated Contractility Drives the Formation of Stress Fibers and Focal Adhesions," Journal of Cell Biology, Vol. 133, No. 6, 1996, pp. 1403-1415. http://dx.doi.org/10.1083/jcb.133.6.1403

[22] N. Wang and D. E. Ingber, "Control of Cytoskeletal Mechanics by Extracellular Matrix, Cell Shape and Mechanical Tension," Biophysical Journal, Vol. 66, No. 6, 1994, pp. 2181-2189.

http://dx.doi.org/10.1016/S0006-3495(94)81014-8

[23] J. M. Maloney, E. B. Walton, C. M. Bruce and K. J. V. Vliet, "Influence of Finite Thickness and Stiffness on Cellular Adhesion-Induced Deformation of Compliant Substrata," Physical Review E, Vol. 78, No. 4, 2008, Article ID: 041923.

http://dx.doi.org/10.1103/PhysRevE.78.041923

[24] S. Sen, A. J. Engler and D. E. Discher, "Matrix Strains Induced by Cells: Computing How Far Cells Can Feel," Cellular and Molecular Bioengineering, Vol. 2, No. 1, 2009, pp. 39-48. http://dx.doi.org/10.1007/s12195-009-0052-Z

[25] J. Solon, I. Levental, K. Sengupta, P. C. Georges and P. A. Janmey, "Fibroblast Adaptation and Stiffness Matching to Soft Elastic Substrates," Biophysical Journal, Vol. 93, No. 12, 2007, pp. 4453-4481. http://dx.doi.org/10.1529/biophysj.106.101386

[26] A. J. Engler, L. Richert, J. Y. Wong, C. Picart and D. E. Discher, "Surface Probe Measurements of the Elasticity of Sectioned Tissue, Thin Gels and Polyelectrolyte Multilayer Films: Correlations between Substrate Stiffness and Cell Adhesion," Surface Science, Vol. 570, No. 1-2, 2004, pp. 142-154. 
http://dx.doi.org/10.1016/j.susc.2004.06.179

[27] J. P. Winer, S. Oake and P. A. Janmey, "Non-Linear Elasticity of Extracellular Matrices Enables Contractile Cells to Communicate Local Position and Orientation," PLoS One, Vol. 4, No. 7, 2009, p. e6382. http://dx.doi.org/10.1371/journal.pone.0006382

[28] S. Y. Tee, J. Fu, C. S. Chen and P. A. Janmey, "Cell Shape and Substrate Rigidity Both Regulate Cell Stiffness," Biophysical Journal, Vol. 100, No. 5, 2011, pp. L25-L27. http://dx.doi.org/10.1016/j.bpj.2010.12.3744

[29] S. Féréol, R. Fodil, B. Labat, S. Galiacy, V. M. Laurent, B. Louis, D. Isabey and E. Planus, "Sensitivity of Alveolar Macrophages to Substrate Mechanical and Adhesive Properties," Cell Motility and the Cytoskeleton, Vol. 63, No. 6, 2006, pp. 321-340. http://dx.doi.org/10.1002/cm.20130

[30] S. Walcott and S. X. Sun, "A Mechanical Model of Actin Stress Fiber Formation and Substrate Elasticity Sensing in Adherent Cells," Proceedings of the National Academy of Sciences USA, Vol. 107, No. 17, 2010, pp. 7757-7762. http://dx.doi.org/10.1073/pnas.0912739107

[31] N. D. Evans, C. Minelli, E. Gentleman, V. LaPointe, S. N. Patankar, M. Kallivretaki, X. Chen, C. J. Roberts and M. M. Stevens, "Substrate Stiffness Affects Differentiation Events in Embryonic Stem Cells," European Cells \& Materials, Vol. 18, 2009, pp. 7188-7192.

[32] J. D. Eshelby, "The Determination of the Elastic Field of an Ellipsoidal Inclusion and Related Problems," Proceedings of the Royal Society of London. Series A, Vol. 241, No. 1226, 1957, pp. 376-396. http://dx.doi.org/10.1098/rspa.1957.0133

[33] A. Zemel and S. A. Safran, "Active Self-Polarization of Contractile Cells in Asymmetrically Shaped Domains," Physical Review E, Vol. 76, 2007, Article ID: 021905. http://dx.doi.org/10.1103/PhysRevE.76.021905

[34] A. Zemel, F. Rehfeldt, A. E. X. Brown, D. E. Discher and S. A. Safran, "Optimal Matrix Rigidity for Stress Fiber Polarization in Stem Cells," Nature Physics, Vol. 6, No. 6, 2010, pp. 468-473. http://dx.doi.org/10.1038/nphys1613

[35] A. Zemel, F. Rehfeldt, A. E. X. Brown, D. E. Discher and S. A. Safran, "Cell Shape, Spreading Symmetry, and the Polarization of Stress-Fibers in Cells," Journal of Physics Condensed Matter, Vol. 22, No. 19, 2010, p. 194110. http://dx.doi.org/10.1088/0953-8984/22/19/194110

[36] A. Nicolas, B. Geiger and S. A. Safran, "Cell Mechanosensitivity Controls the Anisotropy of Focal Adhesions," Proceedings of the National Academy of Sciences of the United States of America, Vol. 101, No. 34, 2004, pp. 12520-12525. http://dx.doi.org/10.1073/pnas.0403539101

[37] T. Shemesh, B. Geiger, A. D. Bershadsky and M. M. Kozlov, "Focal Adhesions as Mechanosensors: A Physical Mechanism," Proceedings of the National Academy of Sciences of the United States of America, Vol. 102, No. 35, 2005, pp. 12383-12388. http://dx.doi.org/10.1073/pnas.0500254102

[38] A. Besser and S. A. Safran, "Force-Induced Adsorption and Anisotropic Growth of Focal Adhesions," Biophysi- cal Journal, Vol. 90, No. 10, 2006, pp. 3469-3484. http://dx.doi.org/10.1529/biophysj.105.074377

[39] V. S. Deshpande, R. M. McMeeking and A. G. Evans, "A Bio-Chemo-Mechanical Model for Cell Contractility," Proceedings of the National Academy of Sciences of the United States of America, Vol. 103, No. 38, 2006, pp. 1401514020. http://dx.doi.org/10.1073/pnas.0605837103

[40] A. Besser and U. S. Schwarz, "Coupling Biochemistry and Mechanics in Cell Adhesion: A Model for Inhomogeneous Stress Fiber Contraction," New Journal of Physics, Vol. 9, No. 425, 2007, pp. 425-441. http://dx.doi.org/10.1088/1367-2630/9/11/425

[41] J. Huang, X. Peng, C. Xiong and J. Fang, "Influence of Substrate Stiffness on Cell-Substrate Interfacial Adhesion and Spreading: A Mechano-Chemical Coupling Model," Journal of Colloid and Interface Science, Vol. 355, No. 2, 2011, pp. 503-308. http://dx.doi.org/10.1016/j.jcis.2010.12.055

[42] R. Merkel, N. Krichgeßner, C. M. Cesa and B. Hoffman, "Cell Force Microscopy on Elastic Layers of Finite Thickness," Biophysical Journal, Vol. 93, No. 9, 2007, pp. 3314-3323. http://dx.doi.org/10.1529/biophysj.107.111328

[43] Y. C. Lin, D. T. Lambe, C. Y. Park, M. R. Wasserman, X. Trepat, R. Krishnan, G. Lenormand, J. F. Fredberg and J. P. Butler, "Mechanosensing of Substrate Thickness," Physical Review E, Vol. 82, No. 4, 2010, Article ID: 041918. http://dx.doi.org/10.1103/PhysRevE.82.041918

[44] A. Buxboim, K. Rajagopal, A. E. X. Brown and D. E. Discher, "How Deeply Cells Feel: Methods for Thin Gels," Journal of Physics: Condensed Matter, Vol. 22, No. 19, 2010, Article ID: 194116. http://dx.doi.org/10.1088/0953-8984/22/19/194116

[45] A. Buxboim, I. L. Ivanovska and D. E. Discher, "Matrix Elasticity, Cytoskeletal Forces and Physics of the Nucleus: How Deeply Do Cells 'Feel' Outside and In?' Journal of Cell Science, Vol. 123, 2010, pp. 297-308. http://dx.doi.org/10.1242/jcs.041186

[46] J. Howard, "Mechanics of Motor Proteins and the Cytoskeleton," Sinauer Associates, Sunderland, 2001.

[47] D. Mitrossilis, J. Fouchard, A. Guiroy, N. Desprat, N. Rodrigues, B. Fabry and A. Asnacios, "Single-Cell Response to Stiffness Exhibits Muscle-Like Behavior," Proceedings of the National Academy of Sciences of the United States of America, Vol. 106, No. 43, 2009, pp. 1824318248. http://dx.doi.org/10.1073/pnas.0903994106

[48] D. Mitrossilis, J. Fouchard, D. Pereira, F. Postic, A. Richert, M. Saint-Jean and A. Asnacios, "Real-Time Single-Cell Response to Stiffness," Proceedings of the National Academy of Sciences of the United States of America, Vol. 107, No. 38, 2010, pp. 16518-16523.

http://dx.doi.org/10.1073/pnas.1007940107

[49] M. Ghibaudo, A. Saez, L. Trichet, A. Xayaphoummine, J. Browaeys, P. Silberzan, A. Buguin and B. Ladoux, "Traction Forces and Rigidity Sensing Regulate Cell Functions," Soft Matter, Vol. 4, No. 9, 2008, pp. 1836-1843. http://dx.doi.org/10.1039/b804103b

[50] M. Balland, N. Desprat, D. Icard, S. Féréol, A. Asnacios, 
J. Browaeys, S. Hénon and F. Gallet, "Power Laws in Microrheology Experiments on Live Cells: Comparative Analysis and Modeling," Physical Review E, Vol. 74, No. 2, 2006, Article ID: 021911.
http://dx.doi.org/10.1103/PhysRevE.74.021911

[51] E. Zamir and B. Geiger, "Molecular Complexity and Dynamics of Cell-Matrix Adhesions," Journal of Cell Science, Vol. 114, 2001, pp. 3583-3590. 